Check for updates

Cite this: RSC Adv., 2018, 8, 31793

Accepted 28th August 2018

DOI: $10.1039 / \mathrm{c} 8 \mathrm{ra06120c}$

rsc.li/rsc-advances
Received 19th July 2018

\section{A double fluorescent nanoprobe based on phosphorus/nitrogen co-doped carbon dots for detecting dichromate ions and dopamine $\uparrow$}

\author{
Bin $\mathrm{Wu}^{\text {ab }}$ Xiaofeng Shi, ${ }^{a}$ Wei Han, ${ }^{\text {a }}$ Taishan Wang, (D) ${ }^{a}$ Chunru Wang (D) ${ }^{a}$ \\ and Li Jiang (D) *ab
}

\begin{abstract}
An "on-off-on" fluorescent phosphorus/nitrogen co-doped carbon dot (PNCD) probe was explored for the determination of $\mathrm{Cr}(\mathrm{VI})$ and dopamine resulting from the inner filter effect (IFE). The blue-emitting carbon dots with high quantum yields of $25.47 \%$ as well as a narrow size distribution were synthesized by a rapid, convenient route using $\mathrm{H}_{3} \mathrm{PO}_{4}$ and ethylenediamine as the precursors without any surface passivation. A wide linear region in the range of $7-70 \mu \mathrm{M}$ with a detection limit of $0.71 \mu \mathrm{M}$ was achieved for $\mathrm{Cr}(\mathrm{V})$. Moreover, the proper reductants can weaken the inner filter effect to recover the PNCD fluorescence by converting $\mathrm{Cr}(\mathrm{VI})$ into $\mathrm{Cr}(\mathrm{III})$. Therefore, the PNCDs/Cr(VI) hybrid could also be used as an "off-on" fluorescent probe for detecting dopamine (DA) with a detection limit of $0.49 \mu \mathrm{M}$. Consequently, the PNCDs could serve as a powerful fluorescent bi-sensor for detection of both $\mathrm{Cr}(\mathrm{VI})$ and DA in practical applications.
\end{abstract}

\section{Introduction}

Carbon dots (CDs) have attracted a great deal of attention because of their fascinating optical, physicochemical and biological properties. ${ }^{1-6}$ Compared with the conventional organic dyes and semiconductor quantum dots, CDs further exhibit some superior properties, which make them attractive in bioimaging, drug delivery, photodynamic therapy, photovoltaic devices, catalysis, energy storage devices and sensing. ${ }^{7-12}$ Moreover, heteroatom doping has been found to effectively improve the photoluminescence properties of CDs. ${ }^{13}$ Nitrogen $(\mathrm{N})$ is considered to be a prominent chemical doping element for tailoring the electronic properties of CDs due to the close similarity of atomic size and strong valence bonds with carbon atoms..$^{14}$ Importantly, C-dots become n-type semiconductors after doping with $\mathrm{N}$ atoms. ${ }^{15}$ Another doping element, phosphorus, can give rise to a substitutional defect in $\mathrm{sp}^{3}$-bonded carbon thin films when CDs act as an n-type donor. ${ }^{16}$ Thus, the electronic characteristics of CDs could be regulated by doping with nitrogen and phosphorus atoms to create more active sites.

In the past decades, heavy-metal ions were constantly released into the environment through industrial and social

${ }^{a}$ CAS Key Laboratory of Molecular Nanostructure and Nanotechnology, CAS Research/Education Center for Excellence in Molecular Sciences, Institute of Chemistry, Chinese Academy of Sciences (CAS), Beijing 100190, P. R. China. E-mail: jiangli@iccas.ac.cn

${ }^{b}$ University of Chinese Academy of Sciences, Beijing 100049, P. R. China

$\dagger$ Electronic supplementary information (ESI) available. See DOI: 10.1039/c8ra06120c activities. Compared to $\mathrm{Cr}(\mathrm{III})$ and $\mathrm{Cr}(0),{ }^{17,18} \mathrm{Cr}(\mathrm{VI})$ is more lifethreatening to community hygiene owing to its vital carcinogenic characteristics. High sensitivity and rapid response are in urgent demand to realize the $\mathrm{Cr}(\mathrm{vI})$ determination at low concentration levels. Although there are a few reports on the successful application of CD-based nanoprobes for $\mathrm{Cr}(\mathrm{vI})$ detection, ${ }^{19,20}$ the important progress in this area is really insufficient.

As a kind of catecholamine neurotransmitter, dopamine (DA), not only can treat depression and be conducive to memory boosting, which contribute to the treatment of Alzheimer's disease, ${ }^{21}$ but also has certain effects on cure of myocardial infraction, hypertension, bronchial asthma and acute heart surgery. ${ }^{22}$ It was worth noting that the abnormal concentration of DA may give rise to patient's hands and feet tremors involuntary or cause Parkinson's disease. ${ }^{23}$ To date, numerous methods for DA detection have been developed, including high performance liquid chromatography ${ }^{24}$ and electrochemical methods. ${ }^{25}$ However, a more effective and sensitive method for DA detection are still in urgent demand.

A dual doped carbon dots was synthesized with a simple onestep route by mixing ethylenediamine (EDA) and phosphoric acid $\left(\mathrm{H}_{3} \mathrm{PO}_{4}\right)$. The carbon dots can be used as a double fluorescent nanoprobe not only to determine the concentration of $\mathrm{Cr}(\mathrm{vI})$ based on the remarkable fluorescence quenching but also to determine DA through a "turn-on" fluorescence recovery. So an "on-off-on" fluorescence system was successfully constructed to be a novel double nanoprobe, which achieved to detect $\mathrm{Cr}(\mathrm{vI})$ and DA synchronously for the first time (Scheme 1). 

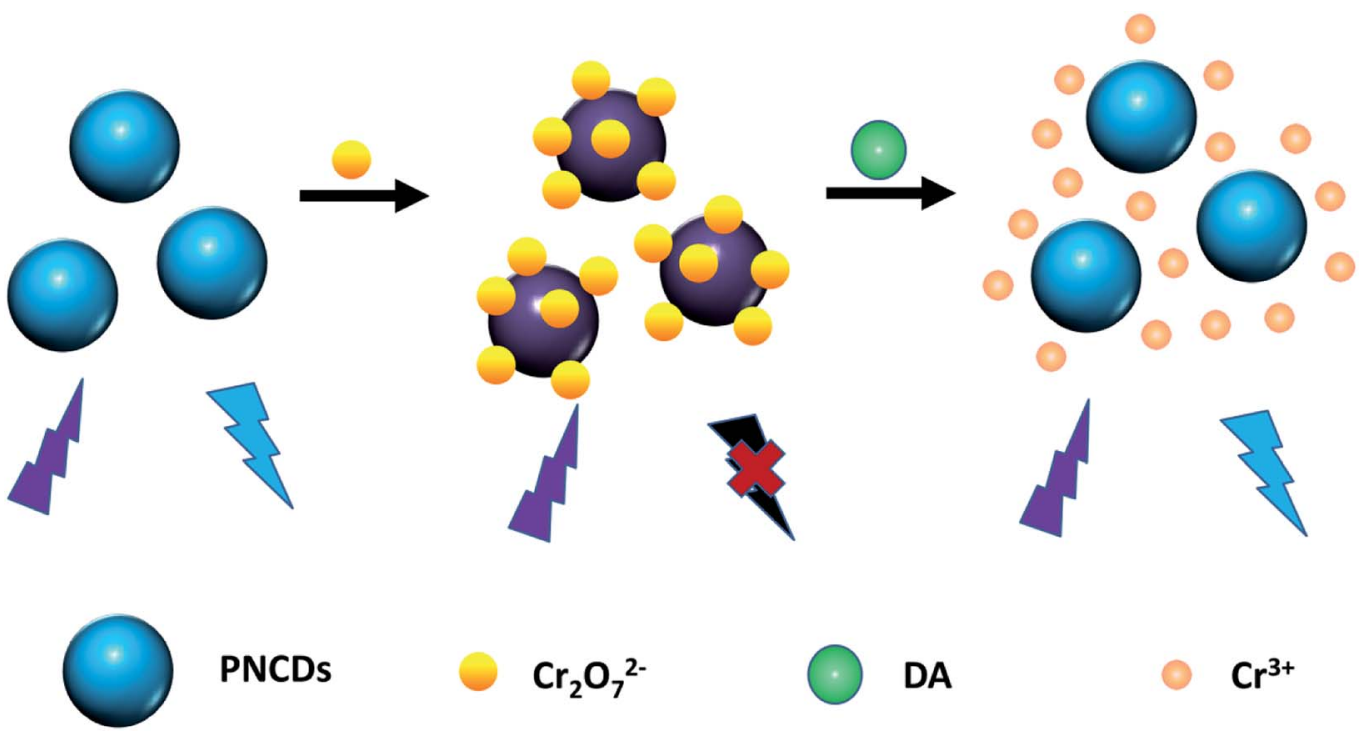

PNCDs

$$
\mathrm{Cr}_{2} \mathrm{O}^{2-}
$$

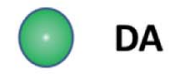

DA

$\mathrm{Cr}^{3+}$

Scheme 1 Diagrammatical explanation of fluorescence mechanism for $\mathrm{Cr}(\mathrm{VI})$ and DA based on IFE of PNCDs.

\section{Experimental section}

\subsection{Materials}

Ethylenediamine (EDA), phosphoric acid $\left(\mathrm{H}_{3} \mathrm{PO}_{4}\right)$ and dopamine (DA) were purchased from Aladdin Ltd. (Shanghai, China, https://www.aladdin-e.com) and used without further purification. All the other reagents were obtained from Sinopharm Chemical Reagent Co., Ltd (Shanghai, China). All the chemicals were of analytical grade. Ultrapure water was used throughout all experiments.

\subsection{Synthesis of phosphorus/nitrogen co-doped carbon dots (PNCDs)}

The homogeneous solutions of ethylenediamine $(1 \mathrm{~mL})$ and $\mathrm{H}_{3} \mathrm{PO}_{4}(0.5 \mathrm{~mL})$ were quickly mixed in a $100 \mathrm{~mL}$ Teflon equipped stainless steel autoclave with vigorous stirring at room temperature. After $30 \mathrm{~min}$, Teflon equipped stainless steel autoclave was placed in oven and heated at $160{ }^{\circ} \mathrm{C}$ for $6 \mathrm{~h}$. After being cooled to room temperature, the products were purified using a $0.22 \mu \mathrm{m}$ filtration membrane to remove nonfluorescent deposits. The filtrate was collected and dialyzed against ultrapure water for $24 \mathrm{~h}$ to remove the residual ethylenediamine and phosphoric acid (dialysis membrane molecular weight cut off $=$ 500 Da, Shanghai Baoman Biological technology Co., Ltd). Finally, PNCDs solution in light brown colour were collected and lyophilized to obtain the dry PNCDs product.

\subsection{Characterization}

The transmission electron microscopic (TEM) images were obtained on a JEOL JEM-2100F transmission electron microscopy (Tokyo, Japan). The X-ray photoelectron spectra (XPS) were recorded on a Kratos AXIS ULTRA DLD X-ray photoelectron spectrometer (Tokyo, Japan). The Fourier transform infrared spectra (FTIR) were acquired on a Bruker Tensor II FTIR spectrometer (Bremen, Germany). The UV-vis was performed on a Lambda 950 absorption spectrophotometer (PerkinElmer,
Llantrisant, UK). Nanosecond fluorescence lifetime assays and photoluminescence spectra were recorded by FLS 980 fluorescence spectrometer steady-state model and time-correlated single-photon counting (TCSPC) system (Edinburgh Instruments Ltd, England), respectively. The quantum yields (QYs) of the as-prepared carbon dots were determined using quinine sulfate in $0.1 \mathrm{M} \mathrm{H}_{2} \mathrm{SO}_{4}$ (literature QYs: $54.6 \%$ ) as the standard sample by comparing the integrated fluorescence intensities (excitation at $360 \mathrm{~nm}$ ) and absorbance values at $360 \mathrm{~nm}$ of the carbon dots aqueous solutions with those of quinine sulfate, which was determined by FLS 980 spectrophotometer. ${ }^{26,27}$

\subsection{Determination of $\mathrm{Cr}(\mathrm{v})$ with PNCDs based fluorescent nanoprobe}

The detection of $\mathrm{Cr}(\mathrm{vI})$ was carried out in aqueous solution at room temperature. In a typical assay, PNCDs powder dissolved in PBS solution $(\mathrm{pH}=7.4)$ and the concentration of PNCDs in solution totally was $0.30 \mathrm{mg} \mathrm{mL} \mathrm{mL}^{-1}$ in $3 \mathrm{~mL}$ bulk volume for quartz cell. Then different volume $\mathrm{Cr}(\mathrm{vI})$ solutions (in PBS) were added into PNCDs solution drop by drop and a series of calculated concentration $(0,0.1,0.2,0.3,0.5,0.7,1,2,3,5,7,10$, $15,20,25,40,50,70,90,100,120,140,160 \mu \mathrm{M})$ were prepared. After solution mixed to equilibrium, the PL spectra were recorded $\left(\lambda_{\text {ex }}=340 \mathrm{~nm}\right.$ ) when PL emission maintained stabilization. A standard curve was fitted using the maximal fluorescence intensity against the $\mathrm{Cr}(\mathrm{vI})$ concentration. According to the standard curve, quantitative detection of $\mathrm{Cr}(\mathrm{vI})$ can be explored. The selectivity of the PNCDs probe towards $\mathrm{Cr}(\mathrm{vI})$ was ascertained by adding other metal ions $\left(\mathrm{K}^{+}, \mathrm{Na}^{+}, \mathrm{Ca}^{2+}, \mathrm{Mg}^{2+}\right.$, $\mathrm{Co}^{2+}, \mathrm{Cu}^{2+}, \mathrm{Ni}^{2+}, \mathrm{Ba}^{2+}, \mathrm{Pb}^{2+}, \mathrm{Fe}^{2+}, \mathrm{Zn}^{2+}, \mathrm{Al}^{3+}, \mathrm{Fe}^{3+}, \mathrm{MnO}_{4}{ }^{-}, \mathrm{ClO}_{4}{ }^{-}$, $\mathrm{ClO}_{3}{ }^{-}$) instead of $\mathrm{Cr}(\mathrm{vI})$ in a same way. The final concentrations of all the metal ions were $100 \mu \mathrm{M}$.

\subsection{Fluorescent detection of DA}

In a typical test, $12 \mu \mathrm{L}$ of the $\mathrm{Cr}(\mathrm{vI})(30 \mathrm{mM})$ was added into $3 \mathrm{~mL}$ of PNCDs solution $\left(0.30 \mathrm{mg} \mathrm{mL} \mathrm{m}^{-1}\right)$ in order to form the probe 
(PNCDs/Cr(vi)). Then, different volume DA solutions (in PBS) was added, and the mixture whose calculated concentration of DA were $0,0.5,1,3,5,7,10,15,20,30,40,50,70,90,100,120$, $140,150,160 \mu \mathrm{M}$ were shaken thoroughly at room temperature, respectively. After $5 \mathrm{~min}$, the fluorescence recovery spectra were measured under the $\lambda_{\text {ex }}$ at $340 \mathrm{~nm}$. The selectivity of the PNCDs/ $\mathrm{Cr}(\mathrm{vI})$ probe towards DA was ensured by adding other reducing biomolecules (fructose, histidine, glucose, $\mathrm{H}_{2} \mathrm{O}_{2}$, urea, glycine, ethylene glycol) instead of DA in a same way. The final concentrations of all reducing biomolecules were $160 \mu \mathrm{M}$.

\section{Results and discussion}

\subsection{Characterization of PNCDs}

Fig. 1a shows the TEM image and size distribution of the PNCDS synthesized at $160{ }^{\circ} \mathrm{C}$. The relatively mono-disperse PNCDs are quasi-spherical with average diameter of $4.14 \mathrm{~nm}$ (the inset images of Fig. 1a). The high-resolution TEM (HRTEM) images (the inset images of Fig. 1a) show that PNCDs sample (PNCDs) possesses the crystalline structure with lattice spacing of $0.21 \mathrm{~nm}$, which are very close to the (100) diffraction planes of graphite. $^{28,29}$ This result indicates that the as-prepared PNCDs possess a graphite-like structure. The corresponding AFM image in tapping mode shown in Fig. S1a $\uparrow$ also indicates that the PNCDs are in the order of $4.1 \mathrm{~nm}$ in diameter and quasispherical shape. The height profile in Fig. $\mathrm{S} 1 \mathrm{~b} \dagger$ reveals that the mean height of the PNCDs is about $2.5 \mathrm{~nm}$.

Chemical and structural information about the PNCDs was further obtained from the FTIR spectra. Fig. $\mathrm{S} 2 \uparrow$ shows the FTIR spectrum of PNCDs. Firstly, a strong and broad peak at $3446 \mathrm{~cm}^{-1}$ for PNCDs indicates the existence of a free $\mathrm{O}-\mathrm{H}$ or
$\mathrm{N}-\mathrm{H}$ stretching vibration, originating in hydroxyl or amine groups on the surface. ${ }^{29,30}$ Peaks at $1570 \mathrm{~cm}^{-1}, 1659 \mathrm{~cm}^{-1}$ and $2860 \mathrm{~cm}^{-1}$ suggest the existence of the $\mathrm{C}=\mathrm{N}$ stretching vibrations, $\mathrm{C}=\mathrm{O}$ or $\mathrm{C}=\mathrm{C}$ stretching vibrations and $\mathrm{C}-\mathrm{H}$ stretching vibrations, respectively. ${ }^{30,31}$ Other characteristic peaks consistent with phosphorus and oxygen-containing groups, such as $\mathrm{P}-$ $\mathrm{OH}$ bending (2471-2782 $\left.\mathrm{cm}^{-1}\right)$, hydrogen-bonded $\mathrm{P}=\mathrm{O}$ (1151 $\left.\mathrm{cm}^{-1}\right)$, P-O-C (aromatic) (1090 $\left.\mathrm{cm}^{-1}\right)$, and $\mathrm{P}-\mathrm{O}-\mathrm{H}$ $\left(970 \mathrm{~cm}^{-1}\right)$ are obtained. ${ }^{31-33}$

The XPS spectra show that the PNCDs samples both contain mainly carbon, nitrogen, phosphorus and oxygen (Fig. 1c), confirming incorporation of $\mathrm{N}$ and $\mathrm{P}$ into the CDs. The C1s peak of PNCDs could be deconvoluted into five peaks (Fig. 1d), corresponding to $\mathrm{C}=\mathrm{C}$ at $284.3 \mathrm{eV}, \mathrm{C}-\mathrm{C}$ at $284.8 \mathrm{eV}, \mathrm{C}-\mathrm{OH} / \mathrm{C}-\mathrm{O}-\mathrm{C}$ at $286 \mathrm{eV}, \mathrm{C}=\mathrm{N}$ at $286.4 \mathrm{eV}$ and $\mathrm{C}=\mathrm{O}$ at $287.9 \mathrm{eV}{ }^{34}$ The $\mathrm{N} 1 \mathrm{~s}$ spectrum shows the characteristic peaks at $399.3 \mathrm{eV}, 400 \mathrm{eV}$ and $400.8 \mathrm{eV}$ are in accord with pyridinic, pyrrolic and graphitic $\mathrm{N}$ (Fig. 1e). ${ }^{35,36}$ The surface components of the PNCDs characterized by XPS are in agreement with the FT-IR analysis, which convictively suggest the successful synthesis of PNCDs.

\subsection{The stability of the as-prepared PNCDs}

When the carbon dots are used for practical sensing applications, good water solubility and fluorescence stability are quite necessary. The prepared PNCDs are inherently water-soluble since there are lots of oxygen-rich functional groups in the structures, as were already proved by the XPS and FT-IR analysis. To investigate the fluorescence stability, the influences of various conditions on the carbon dots were studied (Fig. 2a-d). First, as shown in Fig. 2a, the fluorescence intensities remain
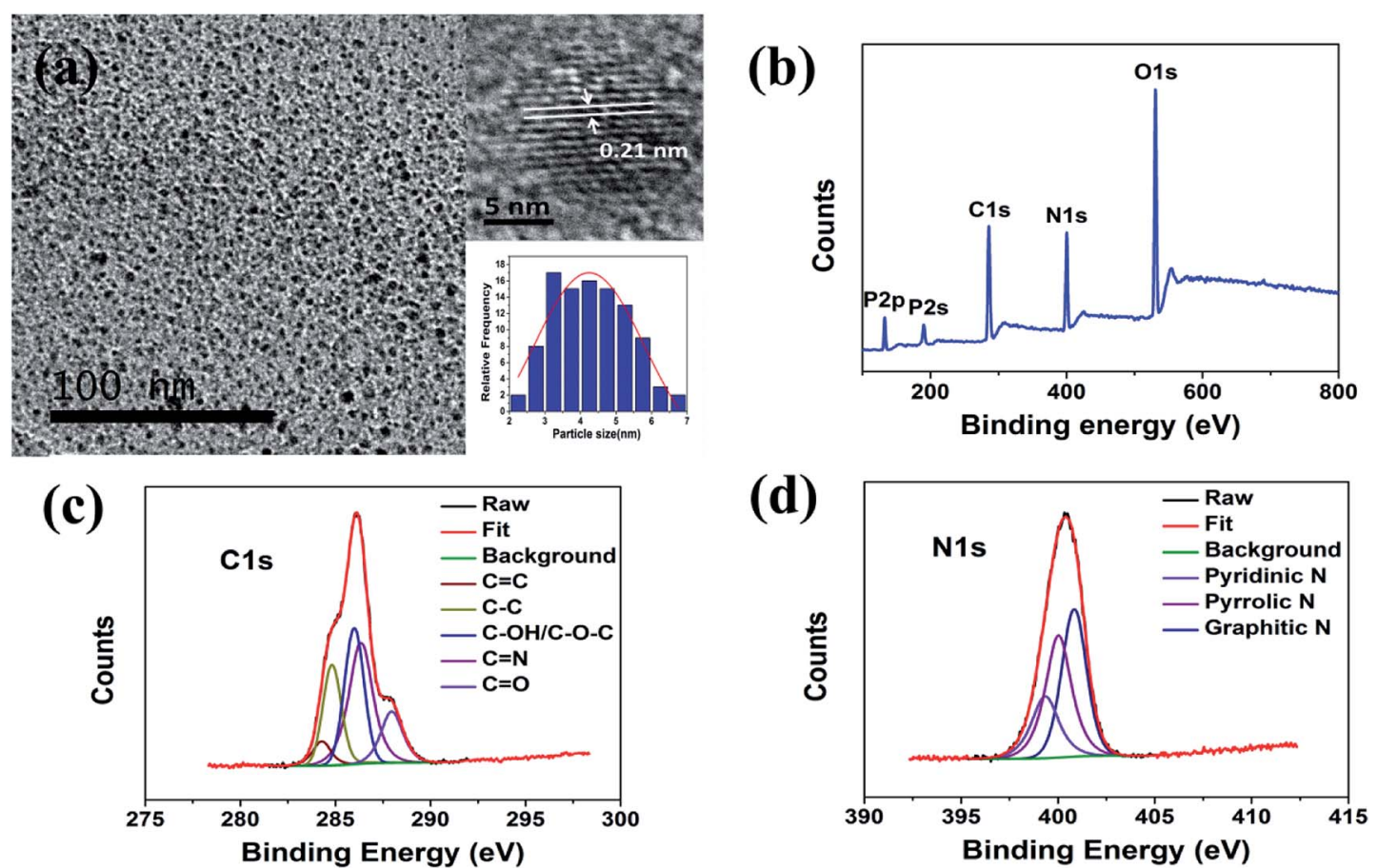

Fig. 1 (a) TEM image of PNCDs; inset images are magnification of a single nanoparticle and particle size distribution histograms of PNCDs. (b) Full-scan XPS spectra of PNCDs. High-resolution XPS spectra of C1s (c) and N1s (d) peaks for PNCDs. 
(a)

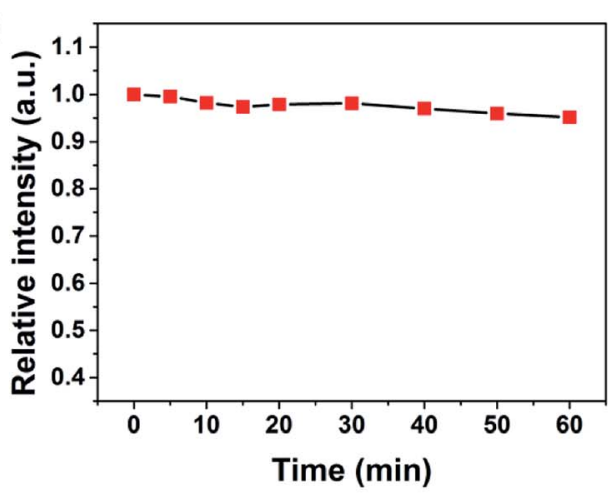

(c)

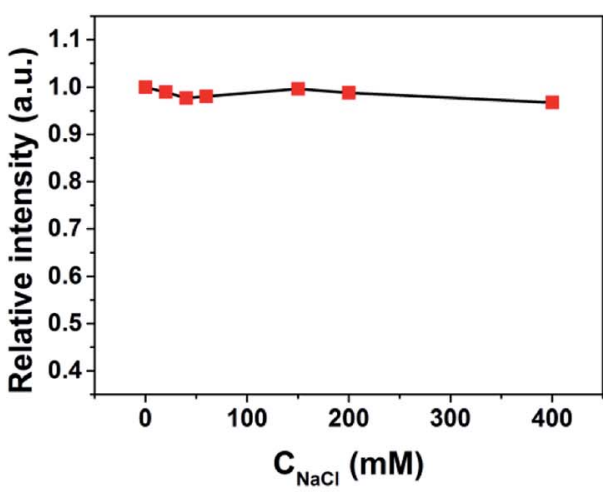

(b)

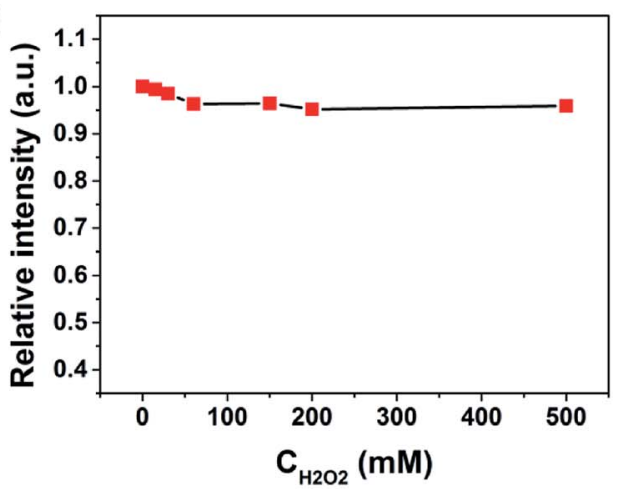

(d)

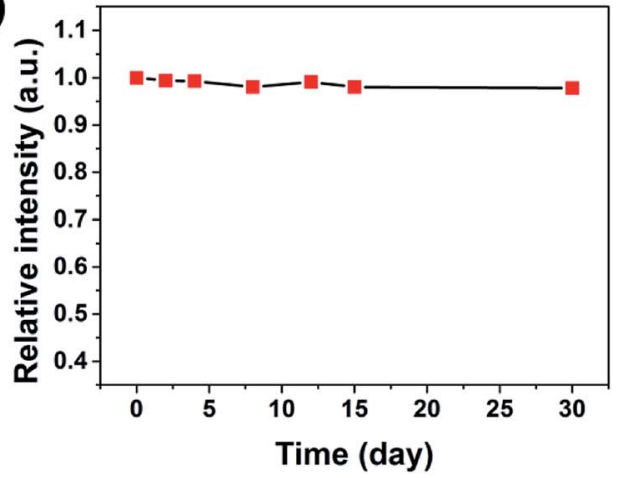

Fig. 2 (a) Effect of time intervals of irradiation with a UV lamp on fluorescence intensity of PNCDs. (b) Effect of oxidant on the fluorescence intensity of PNCDs. (c) Effect of ionic strengths on the fluorescence intensity of PNCDs (ionic strengths were controlled by various concentrations of $\mathrm{NaCl}$ in aqueous solution). (d) Effects of storage time on the fluorescence intensity of the PNCDs.

unchanged under UV irradiation for $60 \mathrm{~min}$, indicating that the PNCDs are highly resistant to photobleaching. Furthermore, the fluorescence intensity of PNCDs does not display significant change after 1 month storage at room temperature (Fig. 2b), showing their excellent photostability. The same phenomenon was also found under the high ionic-strength conditions, the fluorescence intensities keep unchanged with increasing concentrations of $\mathrm{NaCl}$ in aqueous solution (up to $400 \mathrm{mM}$ ) (Fig. 2c). In addition, considering the characteristics of PNCDs being easily oxidized, antioxidant capacity of the PNCDs was explored by changing the concentration of $\mathrm{H}_{2} \mathrm{O}_{2}$ and the result indicated that the PNCDs still possess very strong fluorescence intensity even if the concentration of $\mathrm{H}_{2} \mathrm{O}_{2}$ was higher than $500 \mathrm{mM}$ (Fig. 2d).

\subsection{Optical properties of PNCDs}

To further explore the optical properties of the PNCDs, PL spectra and UV-vis absorption spectra were studied at room temperature. Fig. 3a-c reveals the UV-vis absorption spectra of PNCDs and fluorescence emission spectra of PNCDs. UV-vis absorption spectra of PNCDs shows a weak absorptions peak centered at $270 \mathrm{~nm}$ and a strong absorption peak centered at $330 \mathrm{~nm}$, which are attributed to the $\pi-\pi^{*}$ transition of the conjugated $\mathrm{C}=\mathrm{C}$ band $^{37}$ and the $\mathrm{n}-\pi^{*}$ transition of the $\mathrm{C}=\mathrm{O} /$ $\mathrm{C}=\mathrm{N}$ groups on the surface of the PNCDs, respectively. ${ }^{38}$
In fluorescence spectra, the excitation peak of PNCDs was at $340 \mathrm{~nm}$ and the emission one was found at $420 \mathrm{~nm}$. Furthermore, QYs of the as-fabricated PNCDs was calculated to be $25.47 \%$. Meanwhile, PNCDs also exhibited an excitationdependent PL behavior. With the excitation wavelength changing from 300 to $380 \mathrm{~nm}$, the corresponding PL emission peak shifts from 420 to $442 \mathrm{~nm}$ for the PNCDs. The CIE coordinate in Fig. S3a and $\mathrm{b} \dagger$ reveals the changes in the colour intensity of blue emission with change of excitation wavelengths in the PNCDs solution, which demonstrates all the luminous range of PNCDs synthesized at different temperatures are located at blue area. 3D wall spectra in Fig. 3c further exhibits PNCDs possess excitation-dependent properties, which maximal excitation wavelength is $340 \mathrm{~nm}$. Fig. $3 \mathrm{~d}$ showed the fluorescence spectra of PNCDs, PNCDs-Cr(vI) and PNCDs$\mathrm{Cr}(\mathrm{vI})-\mathrm{DA}$ system. The fluorescence of PNCDs is strong and can be effectively quenched after the addition of $\mathrm{Cr}(\mathrm{vI})$, which is designed as the turn-off nanoprobe. In addition, the enhancement and recovery in the fluorescence can be also observed by the adding of DA as turn-on nanoprobe. This phenomenon is caused by a decrease concentration of $\mathrm{Cr}(\mathrm{vI})$ due to the redox reaction between dopamine and $\mathrm{Cr}(\mathrm{vI})$.

\subsection{Fluorescence response of PNCDs towards $\operatorname{Cr}(\mathrm{vi})$}

The as-fabricated PNCDs could be used as a highly sensitive $\mathrm{Cr}(\mathrm{vI})$ fluorescent probe. Herein, the fluorescence quenching 
(a)

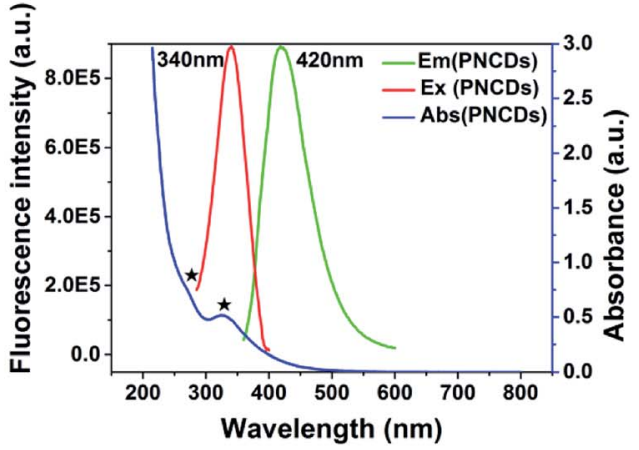

(c)

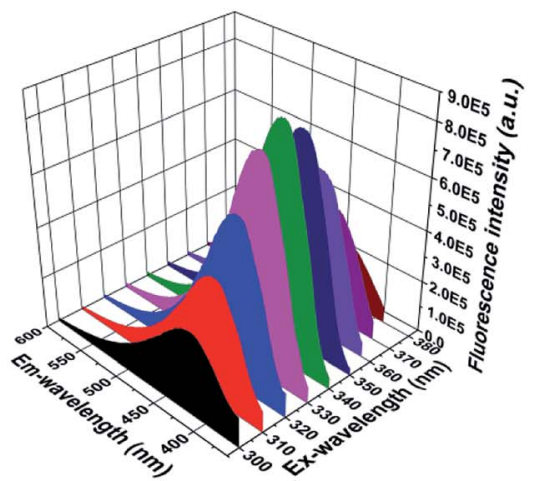

(b)

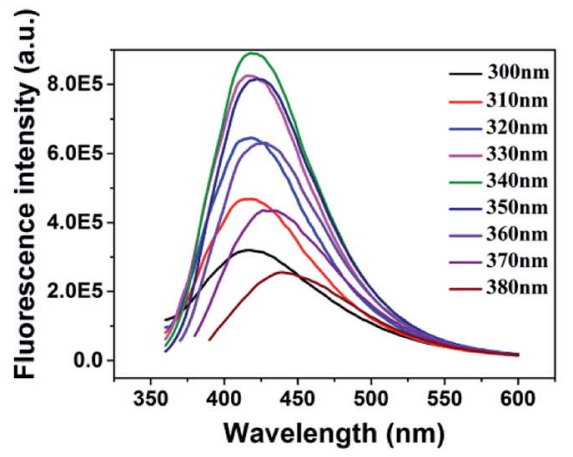

(d)

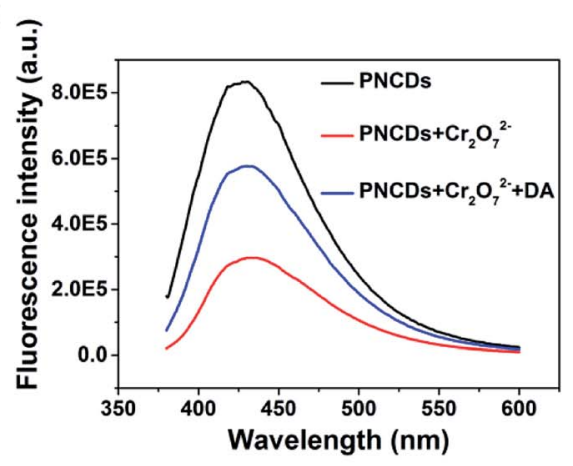

Fig. 3 (a) UV-vis absorption (blue line), PL excitation (red line), and emission (green line) spectra of PNCDs in aqueous solution (0.30 mg mL ${ }^{-1}$ ). (b) Excitation-dependent PL behavior of PNCDs. (c) 3D wall spectra of the PNCDs with different excitation wavelength. (d) Fluorescence emission spectra of PNCDs at different conditions: PNCDs (black line), PNCDs $+\mathrm{Cr}_{2} \mathrm{O}_{7}{ }^{2-}$ (red line), $\mathrm{PNCDs}+\mathrm{Cr}_{2} \mathrm{O}_{7}{ }^{2-}+\mathrm{DA}$ (blue line).

experiments were performed using the PNCDs in the PBS solution $(\mathrm{pH}=7.4)$. A series of fluorescence emission spectra were obtained by increasing the concentration of $\mathrm{Cr}(\mathrm{vI})$ from $0 \mu \mathrm{M}$ to $160 \mu \mathrm{M}$. As shown in Fig. 4a, the fluorescence of PNCDs solution decreased gradually with increase of $\mathrm{Cr}(\mathrm{vI})$ concentration. A linear response was observed between fluorescence intensity quenching ratio $\left(F_{0}-F / F_{0}\right)$ and $\mathrm{Cr}(\mathrm{vI})$ in the range of 7$70 \mu \mathrm{M}$ as shown in Fig. $4 \mathrm{~b}$, where $F_{0}$ and $F$ are the PL intensities of PNCDs at $\lambda_{\text {ex }}$ of $340 \mathrm{~nm}$ without and with $\mathrm{Cr}(\mathrm{vI})$, respectively. It could be expressed as $F_{0}-F / F_{0}=0.11059+0.00494[\mathrm{c}]$ with the value of $R^{2}$ is up to 0.9925 . The limit of detection (LOD) was calculated to be $0.71 \mu \mathrm{M}$ in a similar manner. (LOD $=3 \sigma / S$, where $\sigma$ represents standard deviation of the blank PNCDs sample recorded $(N=11)$ and $S$ is the slope of the calibration curve).

Furthermore, the fluorescence quenching effect of various metal ions on the PNCDs as a probe was investigated (Fig. 4c). For further intuitively figuring out the selectivity of the PNCDs toward $\mathrm{Cr}(\mathrm{vI})$, the potential interfering substances coexisting in samples, including various metal ions $\left(\mathrm{K}^{+}, \mathrm{Na}^{+}, \mathrm{Ca}^{2+}, \mathrm{Mg}^{2+}\right.$, $\mathrm{Co}^{2+}, \mathrm{Cu}^{2+}, \mathrm{Ni}^{2+}, \mathrm{Ba}^{2+}, \mathrm{Pb}^{2+}, \mathrm{Fe}^{2+}, \mathrm{Zn}^{2+}, \mathrm{Al}^{3+}, \mathrm{Fe}^{3+}, \mathrm{MnO}_{4}{ }^{-}, \mathrm{ClO}_{4}{ }^{-}$, $\mathrm{ClO}_{3}{ }^{-}$) were explored. The PL intensity of PNCDs is obviously weakened with addition of $\mathrm{Cr}(\mathrm{vI})$. In contrast, no obvious changes are observed upon addition of other ions into the PNCDs solution. The red bars shown in Fig. 4d represent the fluorescence quenching effect of the PNCDs toward the interfering substances. Contrast experiment were conducted by subsequently adding $100 \mu \mathrm{M} \mathrm{Cr}(\mathrm{vI})$ to each solution (black bars,
Fig. 4d). The results indicate that interferents have no obvious influence on the fluorescence quenching effect of $\mathrm{Cr}(\mathrm{vI})$, which demonstrates excellent anti-interference performance of PNCDs nanoprobe for $\mathrm{Cr}(\mathrm{vI})$ from other ions.

\subsection{Detection of DA by PNCDs/Cr(vI) nanoprobe}

$\mathrm{Cr}(\mathrm{vI})$ can be reduced by DA under mild circumstances (normal temperature, in neutral environment). ${ }^{39,40}$ In Fig. 5a, the fluorescence of PNCDs solution enhanced with the concentration of DA ranging from $0 \mu \mathrm{M}$ to $160 \mu \mathrm{M}$. A linear response is observed between fluorescence intensity quenching ratio $\left(F_{0}-F\right) / F_{0}$ and DA in the range of $1-50 \mu \mathrm{M}$ as shown in Fig. 5b. It could be expressed as $F_{0}-F / F_{0}=-0.14351+0.00418[\mathrm{c}]$ with the value of $R^{2}$ is up to 0.9936 . The limit of detection (LOD) was calculated to be $0.49 \mu \mathrm{M}$ in a similar manner. (LOD $=3 \sigma / S$, where $\sigma$ is represents standard deviation of the PNCDs sample recorded $(N$ $=10$ ) and $S$ is the slope of the calibration curve). For further investigating the selectivity of the PNCDs/Cr(vI) towards DA, the potential interfering substances coexisting in samples, including various reducing biomolecules (His, Glu, Gly, urea, Flu, $\mathrm{H}_{2} \mathrm{O}_{2}$, ethylene glycol, DA) were explored. In this test, the effects of reducing biomolecules on the PNCDs/Cr(vi) fluorescence recovery under the same conditions were displayed in Fig. 5c. It is found that among all the reducing biomolecules examined, DA is the only one that can restore the fluorescence of PNCDs/Cr(vI), indicating that the "off-on" detection is specific to DA. For PNCDs/Cr(vI) system, $\mathrm{Cr}(\mathrm{vI})$ may be stably 
(a)

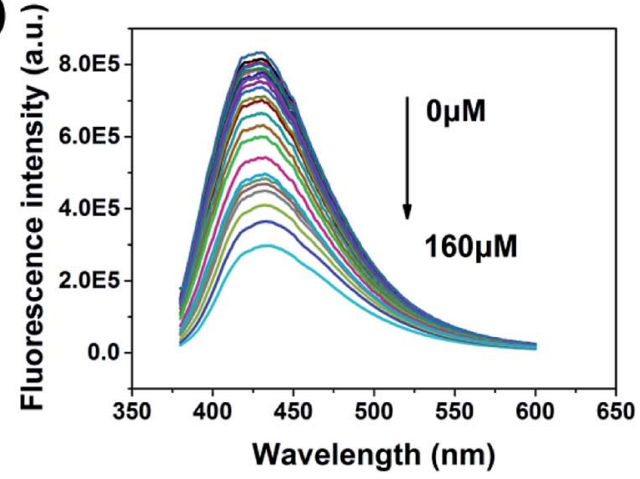

(c)

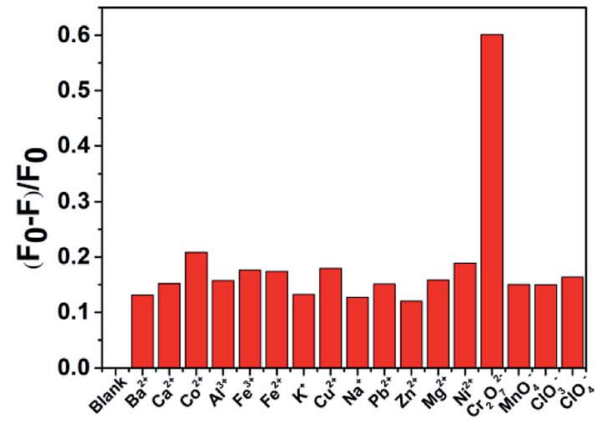

(b)

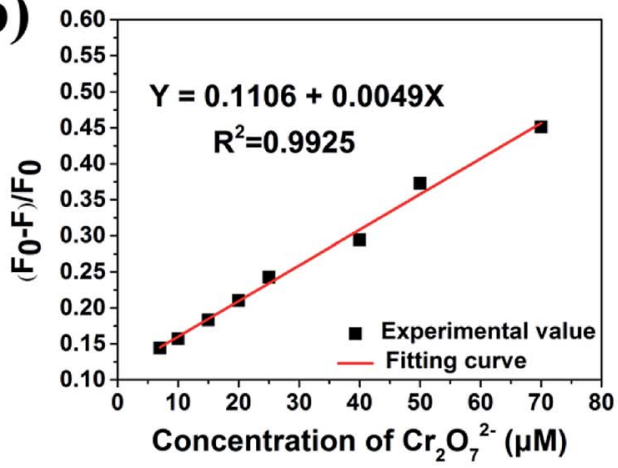

(d)

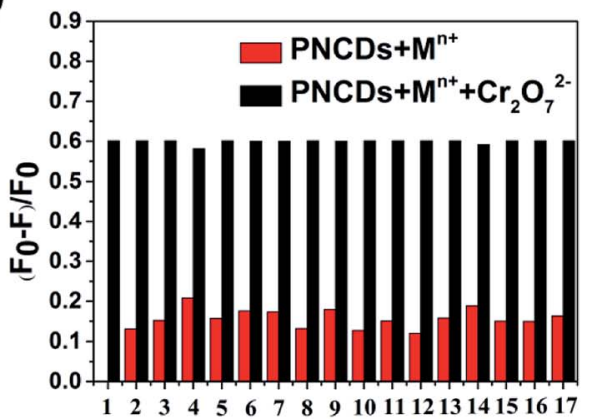

Fig. 4 (a) The fluorescence responses of the PNCDs in PBS ( $\mathrm{pH}: 7.4)$ after the addition of different concentrations of $\mathrm{Cr}(\mathrm{vl})(0,0.1,0.2,0.3,0.5$, $\left.0.7,1,2,3,5,7,10,15,20,25,40,50,70,90,100,120,140,160 \mu \mathrm{mol} \mathrm{L}^{-1}\right)$. (b) Plot of the $\left(F_{0}-F\right) / F_{0}$ with the concentration of $C r(\mathrm{vI})$. (c) and (d) $\left(F_{0}-\right.$ $F) / F_{0}$ of the PNCDs in PBS (pH: 7.4) with $100 \mu \mathrm{M}$ different metal ions. $F$ and $F_{0}$ correspond to the fluorescence intensities of the PNCDs with and without $100 \mu \mathrm{M}$ of different metal ions (the numbers 1 to 17 are corresponding to blank, $\mathrm{Ba}^{2+}, \mathrm{Ca}^{2+}, \mathrm{Co}^{2+}, \mathrm{Al}^{3+}, \mathrm{Fe}^{3+}, \mathrm{Fe}^{2+}, \mathrm{K}^{+}, \mathrm{Cu}^{2+}, \mathrm{Na}^{+}, \mathrm{Pb}^{2+}$, $\mathrm{Zn}^{2+}, \mathrm{Mg}^{2+}, \mathrm{Ni}^{2+}, \mathrm{MnO}_{4}^{-}, \mathrm{ClO}_{3}^{-}, \mathrm{ClO}_{4}^{-}$, respectively).

reduced to $\mathrm{Cr}(\mathrm{III})$ by DA, leading to eliminating of IFE effect resulting from the reduction of $\mathrm{Cr}(\mathrm{VI})$ into $\mathrm{Cr}(\mathrm{III})$ recovered the fluorescence of PNCDs. Consequently, this PNCDs/Cr(vi) system could be utilized as a"turn-on" fluorescence probe for DA determination. The red bars shown in Fig. $5 \mathrm{~d}$ represent the fluorescence recovery effect of the PNCDs/Cr(vi) toward the interfering substances. Contrast experiments were performed by subsequently adding $160 \mu \mathrm{M}$ DA to each solution (black bars, Fig. 5d). The results indicate that interferents have no obvious influence on the fluorescence recovery effect of DA, which demonstrates excellent anti-interference performance of PNCDs/Cr(vi)/DA nanoprobe from other reducing biomolecules. Additionally, the effects of other reducing agents, including ascorbic acid, $\mathrm{Na}_{2} \mathrm{~S}$ and $\mathrm{NaSO}_{3}$, on the PNCDs/Cr(vI) fluorescence recovery under the same above-described conditions were displayed in Fig. S4. $\uparrow$ The results showed that all above reducing substances can restore the fluorescence of PNCDs-Cr(vI) to some extent except ethylene glycol. However, the restoration of dopamine is obviously superior to the others as shown in Fig. S4. $\dagger$ So it is believed that this PNCDs-Cr(vi) system still detected dopamine effectively.

Moreover, the effects of the analogues of dopamine, epinephrine (EP) and noradrenaline (NE), on the PNCDs/Cr(vI) fluorescence recovery under the same above-described conditions were also investigated as shown in Fig. S5. $\uparrow$ The results showed both EP and NE had almost the same response to fluorescence restoration with dopamine. As the analogues of dopamine, EP and NE have the same ortho-phenol hydroxyl as functional groups reacting with $\mathrm{Cr}(\mathrm{vI})$, so the similar phenomenon of fluorescence restoration is believed to be reasonable. Their ortho-phenol hydroxyl could be oxidized as ketone carbonyl to restore the fluorescence.

\subsection{Construction of fluorescence switch}

The fluorescence switch property of PNCDs based on the control of $\mathrm{Cr}(\mathrm{VI})$ and DA was investigated. As shown in Fig. 6, the fluorescence of PNCDs could dramatically decreased by $160 \mu \mathrm{M}$ $\mathrm{Cr}(\mathrm{vI})$, subsequently, the quenchable fluorescence could be restored by $160 \mu \mathrm{M}$ DA due to the strong redox reaction between $\mathrm{DA}$ and $\mathrm{Cr}(\mathrm{vr})$, then the fluorescence of system was quenched again by adding $\mathrm{Cr}(\mathrm{vI})$ and so on. The fluorescence intensity of PNCDs was regulated by alternate addition of increasing $\mathrm{Cr}(\mathrm{vI})$ and DA for four times, implying the relative excellent fluorescence switch character of PNCDs.

\subsection{Mechanism of PNCDs quenching}

A series of experiments were performed to figure out the PL quenching mechanism of PNCDs. The spectral overlapping between the absorption band of the $\mathrm{Cr}(\mathrm{vI})$ and the excitation or 
(a)

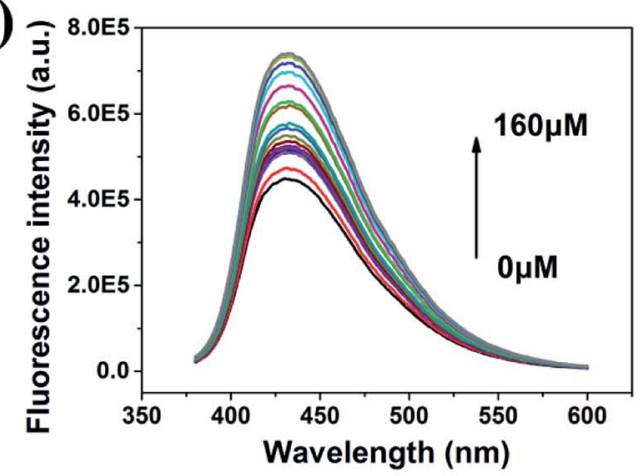

(c)

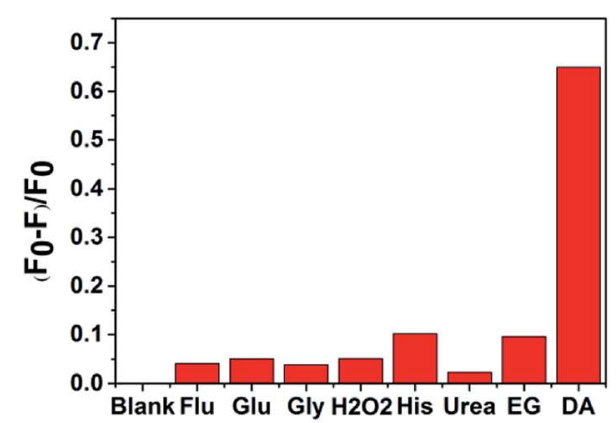

(b)

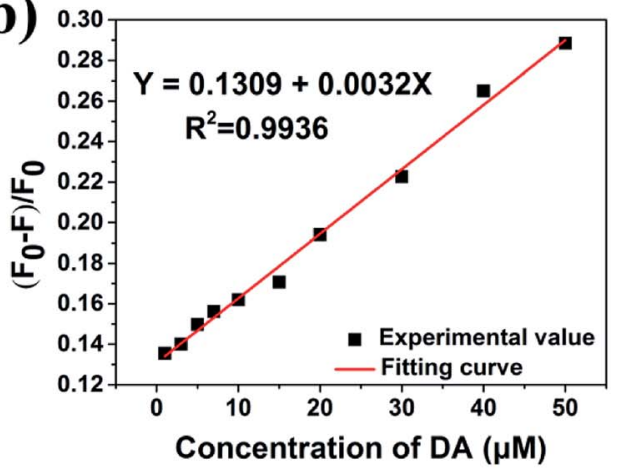

(d)

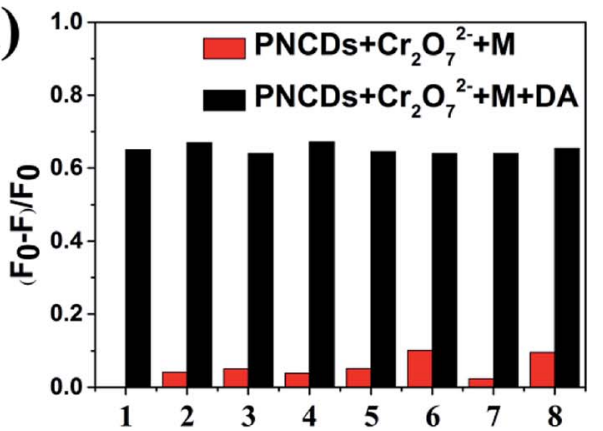

Fig. 5 (a) Fluorescence recovery of the PNCDs/Cr(vı) mixture to DA at various concentrations $(0,0.5,1,3,5,7,10,15,20,30,40,50,70,90,100$, $\left.120,140,150,160 \mu \mathrm{mol} \mathrm{L}^{-1}\right)$. (b) Plot of the $\left(F_{0}-F\right) / F_{0}$ with the concentration of DA. (c) and (d) $\left(F_{0}-F\right) / F_{0}$ of the PNCDs in PBS (pH: 7.4$)$ with 160 $\mu \mathrm{M}$ different reducing biomolecules. $F$ and $F_{0}$ correspond to the fluorescence intensities of the PNCDs without and with $160 \mu M$ of different reducing biomolecules (the numbers 1 to 8 are corresponding to blank, Flu, Glu, Gly, $\mathrm{H}_{2} \mathrm{O}_{2}$, His, urea, EG, respectively).

emission band of the PNCDs shown in Fig. 3a suggested that the fluorescence modulation be related to the energy transfer, IFE process or electron transfer. ${ }^{\mathbf{4 1}-43}$ All the decay curves of the PNCDs in the absence and presence of $120 \mu \mathrm{M} \mathrm{Cr}(\mathrm{vI})$ and PNCDs/Cr(vi)/DA mixture followed third order exponential decay (Fig. 7b). Their calculated average lifetimes were found to be $8.31 \mathrm{~ns}, 8.24 \mathrm{~ns}$ and $8.27 \mathrm{~ns}$, respectively. These unchanged

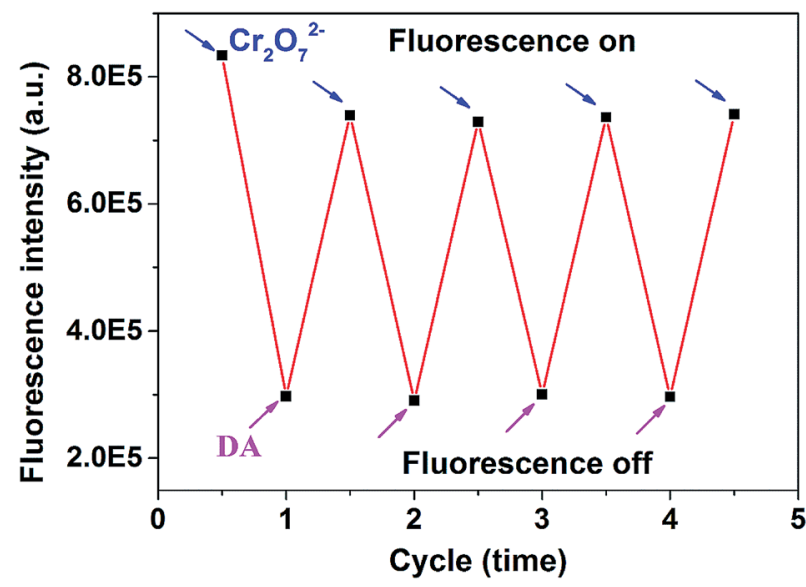

Fig. 6 Fluorescence intensity changes at $340 \mathrm{~nm}$ of PNCDs upon alternate addition of concentrations of $160 \mu \mathrm{M} \mathrm{Cr}(\mathrm{vI})$ and $160 \mu \mathrm{M}$ DA in PBS (pH: 7.4). lifetimes showed that no energy transfer happened between PNCDs and $\mathrm{Cr}(\mathrm{vI}) .^{\mathbf{4 4}}$

Cyclic voltammetry (CV) was used to evaluate the Highest Occupied Molecular Orbital (HOMO) and Lowest Unoccupied Molecular Orbital (LUMO) energy levels of PNCDs by using a standard three-electrodes system, in which of glassy carbon worked as the working electrode with a Pt-wire counter electrode and an $\mathrm{Ag} / \mathrm{AgCl}$ reference electrode. By using cyclic voltammetry with the potential sweeping between -1.5 and $1.0 \mathrm{~V}$ at a scan rate of $0.1 \mathrm{~V} \mathrm{~s}^{-1}$, ECL signals of the PNCDs $\left(0.3 \mathrm{mg} \mathrm{mL}^{-1}\right)$ were recorded in DMF containing $0.1 \mathrm{M}(\mathrm{Bu}){ }_{4} \mathrm{NBF}_{4}$ as the supporting electrolyte. The reduction potential for PNCDs was determined to be $-0.37 \mathrm{eV}$ (Fig. 7c). The HOMO and LUMO energy levels of PNCDs are calculated according to the following equations: ${ }^{45}$

$$
\begin{gathered}
E_{\mathrm{LUMO}}=-\mathrm{e}\left(E_{\mathrm{Red}}+4.4\right) \\
E_{\mathrm{HOMO}}=-\mathrm{e}\left(E_{\mathrm{Ox}}+4.4\right) \\
E_{\mathrm{HOMO}}=E_{\mathrm{LUMO}}-E_{\mathrm{g}}
\end{gathered}
$$

where $E_{\mathrm{Ox}}$ and $E_{\text {Red }}$ are the initial value of oxidation and reduction potential for PNCDs, respectively. So the corresponding LUMO level is $-4.03 \mathrm{eV}$. The HOMO energy could not be obtained due to the irreversible of the oxidation behaviour. To determine the HOMO levels of PNCDs, we combine $E_{\text {Red }}$ with 
(a) $\bar{j}$

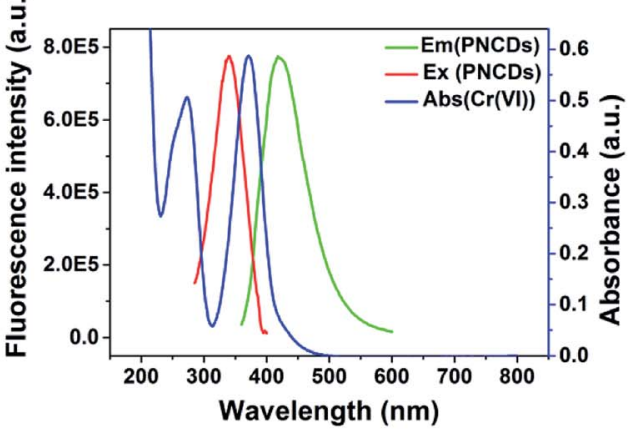

(c)

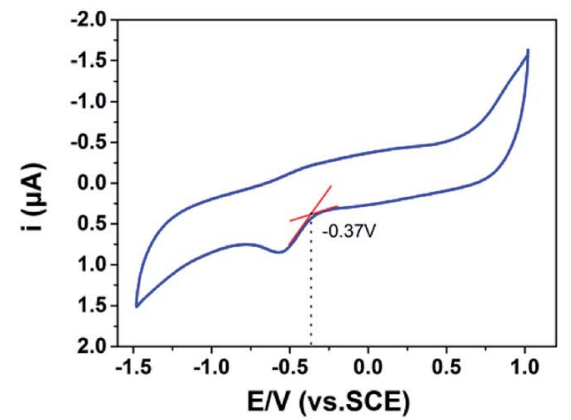

(b)

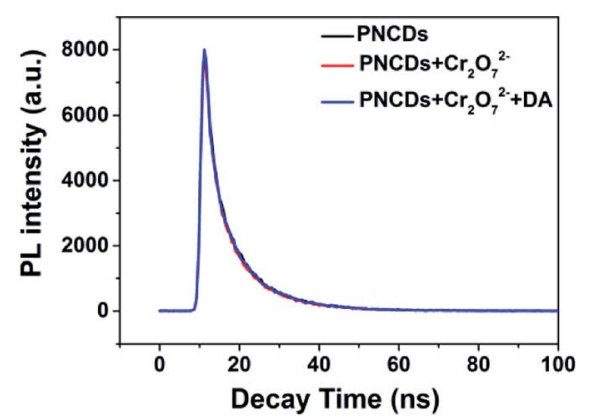

(d)

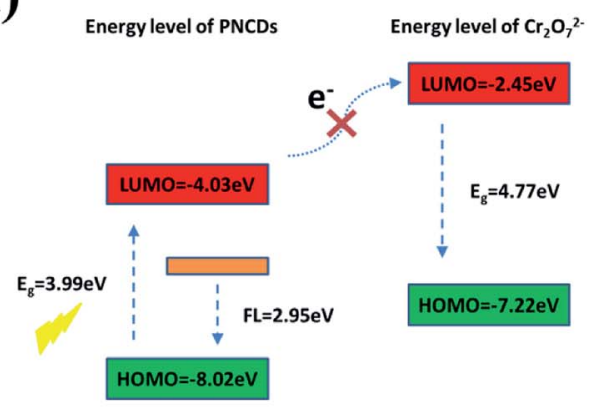

Fig. 7 (a) UV-vis absorption spectra of $\mathrm{Cr}(\mathrm{VI})$ and the excitation and emission spectra of PNCDs. (b) The time-correlated single-photon counting (TCSPC) of PNCDs alone (black line), PNCDs with Cr(vI). (c) The cyclic voltammetry curve of PNCDs. (d) Energy levels of the LUMO and HOMO of PNCDs and $\mathrm{Cr}(\mathrm{VI})$.

the optical energy band gap $\left(E_{\mathrm{g}}\right.$, resulting from the absorption edge in the absorption spectrum), which is estimated to be $3.99 \mathrm{eV}$. So the $E_{\text {Hомо }}$ of PNCDs is calculated to be $-8.02 \mathrm{eV}$. Meanwhile, the $E_{\mathrm{LUMO}}$ and $E_{\mathrm{HOMO}}$ of $\mathrm{Cr}(\mathrm{vI})$ could be calculated as $-2.45 \mathrm{eV}$ and $-7.22 \mathrm{eV}$ obtained from DFT calculations. Apparently, as shown in Fig. 7d, it is impossible for the electron in the LUMO of the PNCDs to transfer to the LUMO of the $\mathrm{Cr}(\mathrm{vI})$.

Moreover, the Stern-Volmer's model ${ }^{44}$ was used to model the quenching kinetics of PNCDs with $\mathrm{Cr}(\mathrm{VI})$.

$$
F_{0} / F=1+K_{\mathrm{sv}}[\mathrm{c}]=1+K_{\mathrm{q}} \tau_{0}[\mathrm{c}]
$$

where $F_{0}$ and $F$ are the fluorescence intensities of the PNCDs in the absence and in the presence of a quencher respectively. [c] is the concentration of the quencher, $K_{\mathrm{sv}}$ is the dynamic quenching constant, and in this work $K_{\mathrm{sv}}=0.00494 \mu \mathrm{M}^{-1}$ by taking the slope of the regression line in Fig. $4 \mathrm{~b}$ according to the regression analysis above, $K_{\mathrm{q}}$ is the quenching rate constant and $\tau_{0}$ is the fluorescence lifetime of PNCDs ( $\left.\tau_{0}=8.31 \mathrm{~ns}\right)$. The $K_{\mathrm{q}}$ of $5.95 \times 10^{11} \mathrm{M}^{-1} \mathrm{~s}^{-1}$, suggesting a static quenching rather than a dynamic quenching in accordance with the rate constant of a dynamic quenching being less than $1.0 \times 10^{10} \mathrm{M}^{-1} \mathrm{~s}^{-1}$, confirmed IFE mechanism..$^{44,46,47}$ The UV-vis spectra of the PNCDs, PNCDs/Cr(vI), and $\mathrm{Cr}(\mathrm{vI})$, shown in Fig. S6a, $\uparrow$ which suggested IFE mechanism is the only possible dominated reason for the fluorescence quenching of PNCDs. Additionally, absorption changes of PNCDs with increasing concentrations of $\mathrm{Cr}(\mathrm{VI})$ for UV-vis spectra is showed in Fig. S6b. $\dagger$ The result also demonstrated the rationality of IFE based fluorescence quenching.
Since both the energy transfer and electron transfer process are excluded, the IFE appears to be more favoured for sufficient spectral overlaps between the absorption band of the $\mathrm{Cr}(\mathrm{VI})$ and the excitation and emission band of the PNCDs.

Based on the significant fluorescence restoration of the PNCDs/Cr(vI) system in the simultaneous presence of DA, an molecular logic gate was designed with this nanoprobe (Fig. 8). In this logic gate, $\mathrm{Cr}(\mathrm{VI})$ and $\mathrm{DA}$ were appointed as inputs along

(a)

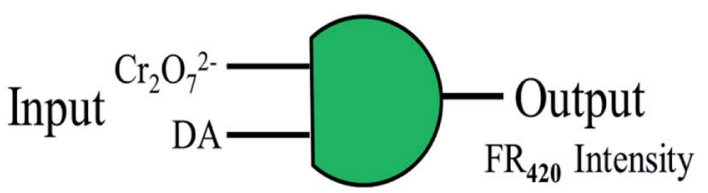

(b)

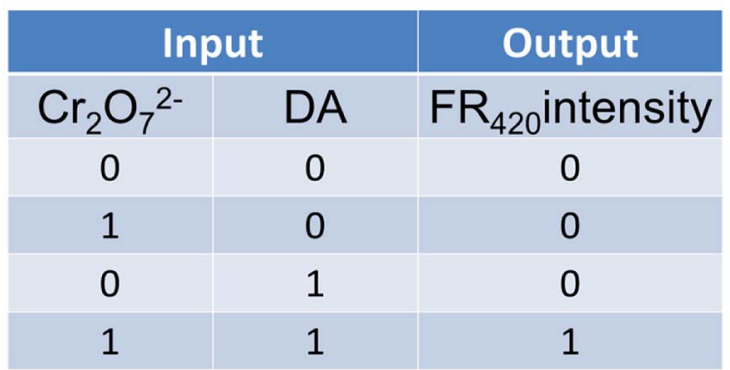

Fig. 8 (a) The combinatorial logic scheme of "AND" logic operations and (b) truth table. 
Table 1 Comparison of detection performance of different fluorescent probes for $\mathrm{Cr}(\mathrm{vI})$ detection

\begin{tabular}{llll}
\hline Materials & LOD & Linear range & Reference \\
\hline N, S-co-doped carbon dots & $0.52 \mu \mathrm{M}$ & $1-40 \mu \mathrm{M}$ & 48 \\
Co(II)-doped carbon dots & $1.17 \mu \mathrm{M}$ & $5-125 \mu \mathrm{M}$ & 49 \\
P, N-co-doped carbon dots & $23 \mathrm{nM}$ & $1.5-30 \mu \mathrm{M}$ & 50 \\
P, N-co-doped carbon dots & $0.48 \mu \mathrm{M}$ & $1.6-12 \mu \mathrm{M}$ & 51 \\
P, N-co-doped carbon dots & $0.71 \mu \mathrm{M}$ & $7-70 \mu \mathrm{M}$ & This work
\end{tabular}

Table 2 Comparison of detection performance of different fluorescent probes for DA detection

\begin{tabular}{llll}
\hline Materials & LOD & Linear range & Reference \\
\hline GO & $94 \mathrm{nM}$ & $0.25-20 \mu \mathrm{M}$ & 52 \\
CNPs/Fe ${ }^{3+}$ & $68 \mathrm{nM}$ & $0.1-10 \mu \mathrm{M}$ & 53 \\
ZnO QDs & $12 \mathrm{nM}$ & $0.05-10 \mu \mathrm{M}$ & 54 \\
P, N-co-doped carbon dots & $0.49 \mu \mathrm{M}$ & $1-50 \mu \mathrm{M}$ & This work
\end{tabular}

with the fluorescent state of "off" and "on" and restored FL intensity at $420 \mathrm{~nm}$ taken as the output. As for input in truth table (Fig. 8a), the presence and absence of $\mathrm{Cr}(\mathrm{VI})$ and DA are assigned as " 1 " and " 0 ", respectively. For output, we define the strong FL change as " 1 " and weak FL change as "0". The output is " 0 " with the absence of inputs $(0 / 0)$ of them and $\mathrm{Cr}(\mathrm{vI})(1,0)$ or DA $(0,1)$ input alone. Inversely, only in the existence of both $\operatorname{Cr}(\mathrm{vI})$ and DA $(1,1)$, output is activated and offers an output signal of " 1 ". It provides thoughts about design of molecular based logic device for peers with "on-off-on" nanoprobe. Meanwhile, we investigated another application of PNCDs as a fluorescent ink for display purposes in Fig. $\mathrm{S} 7 \dagger$ and experimental result showed that PNCDs ink is clear, permanent, pollution free, and easily washable. Therefore, PNCDs ink might be an alternative and potential for fluorescent pens.

Finally, we compared this work with the reported work as shown in Tables 1 and 2. In Table 1 for detecting Cr(vi), PNCDs in our work showed obviously wider linear range though the detection limit was inferior to the others. But it is believed that wider linear range could broaden practical applications in detection of water and soil pollutants. In the same way, PNCDs$\mathrm{Cr}(\mathrm{vI})$ system could detect DA with a much wider linear range compared to the previous works listed in Table 2, which displayed more practical application prospect.

\section{Conclusions}

In this work, the phosphorus and nitrogen dual-doped CDs with strong blue fluorescence have been fabricated by a rapid, convenient route with $\mathrm{H}_{3} \mathrm{PO}_{4}$ and ethylenediamine as the precursors without any surface passivation. The quantum yield of PNCDs reaches as high as $25.47 \%$. The fundamentals of a simple and efficient "on-off-on" fluorescence technique for determination of $\mathrm{Cr}(\mathrm{vI})$ and DA utilizing PNCQDs as a fluorescent nanoprobe based on IFE have been illustrated. The fluorescence quenching of the as-fabricated carbon dots was observed with the increasing $\mathrm{Cr}(\mathrm{vI})$ concentration, and the calibration curve displayed a wide linear region over the range of 7-70 $\mu \mathrm{M}$ with a detection limit of $0.71 \mu \mathrm{M}$. Meanwhile, the fluorescence recovery ratio and the concentration of DA have a linear relationship in the range of $1-50 \mu \mathrm{M}$ with a detection limit of $0.49 \mu \mathrm{M}$. With high sensitivity, good selectivity and wide linear range, PNCDs may be well applied in chemical and biological systems in future.

\section{Conflicts of interest}

There are no conflicts to declare.

\section{Acknowledgements}

The authors thank the financial support by the National Natural Science Foundation of China (Grant No. 21673257, 61227902, 51472248, and 51672281).

\section{Notes and references}

1 S. N. Baker and G. A. Baker, Angew. Chem., Int. Ed., 2010, 49, 6726-6744.

2 H. Li, Z. Kang, Y. Liu and S. T. Lee, J. Mater. Chem., 2012, 22, 24230-24253.

3 S. Y. Lim, W. Shen and Z. Gao, Chem. Soc. Rev., 2015, 44, 362381.

4 A. Kumar, A. R. Chowdhuri, D. Laha, S. Chandra, P. Karmakar and S. K. Sahu, RSC Adv., 2016, 6, 58979-58987.

5 S. H. Wang, H. Y. Niu, S. J. He and Y. Q. Cai, RSC Adv., 2016, 6, 107717-107722.

6 K. Duan, J. Weng and J. X. Wang, RSC Adv., 2015, 5, 9024590254.

7 W. Zhao, Y. Li, S. Yang, Y. Chen, J. Zheng, C. Liu, Z. Qing, J. Li and R. Yang, Anal. Chem., 2016, 88, 4833-4840.

8 N. Li, A. Tan, X. Wang, S. Xu, L. Sun, H. Duan, C. Xu and P. Chen, ACS Nano, 2016, 10, 3622-3629.

9 L. Lin, X. Song, Y. Chen, M. Rong, T. Zhao, Y. Wang, Y. Jiang and X. Chen, Anal. Chim. Acta, 2015, 869, 89-95.

10 J. Shen, Y. Zhu, X. Yang and C. Li, Chem. Commun., 2012, 48, 3686-3699.

11 X. J. Zheng, R. P. Liang, Z. J. Li, L. Zhang and J. D. Qiu, Sens. Actuators, B, 2016, 230, 314-319.

12 X. T. Zheng, A. Tan, A. Ananthanaraya, D. H. Kim and P. Chen, ACS Nano, 2013, 7, 62-78.

13 Y. Du and S. Guo, Nanoscale, 2016, 8, 25-32.

14 S. U. Lee, R. V. Belosludov, H. Mizuseki and Y. Kawazoe, Small, 2009, 5, 1769-1775.

15 D. Wei, Y. Liu, Y. Wang, H. Zhang, L. Huang and G. Yu, Nano Lett., 2009, 9, 1752-1758.

16 J. Zhou, X. Shan, J. Ma, Y. Gu, Z. Qian, J. Chen and H. Feng, RSC Adv., 2014, 4, 5465-5468.

17 A. Baral and R. D. Engelken, Environ. Sci. Policy, 2002, 529, 47-51.

18 A. Zhitkovich, Chem. Res. Toxicol., 2011, 24, 1617-1629.

19 M. Zheng, Z. Xie, D. Qu, D. Li, P. Du, X. Jing and Z. Sun, ACS Appl. Mater. Interfaces, 2013, 5, 13242-13247. 
20 M. Hosseini, V. K. Gupta, M. R. Ganjali, Z. Rafiei-Sarmazdeh, F. Faridbod, H. Goldooz, A. R. Badiei and P. Norouzi, Anal. Chim. Acta, 2012, 715, 80-85.

21 A. Roychoudhury, S. Basu and S. K. Jha, Biosens. Bioelectron., 2016, 84, 72-81.

22 H. Beitollahi, H. Karmi-Maleh and H. Khabazzadeh, Anal. Chem., 2008, 80, 9848-9851.

23 J. Lotharius and P. Brundin, Nat. Rev. Neurosci., 2002, 3, 932942.

24 K. Syslova, L. Rambousek, M. Kuzma, V. Najmanova, V. Bubenikova-Valesova, R. Slamberova and P. Kacer, J. Chromatogr. A, 2011, 1218, 3382-3391.

25 S. R. Hu, Q. T. Huang, Y. Lin, C. Wei, H. Q. Zhang, W. X. Zhang, Z. B. Guo, X. X. Bao, J. G. Shi and A. Y. Hao, Electrochim. Acta, 2014, 130, 805-809.

26 X. Liu, X. Tang, Y. Hou, Q. Wu and G. Zhang, RSC Adv., 2015, 5, 81713-81722.

27 H. Zheng, Q. Wang, Y. Long, H. Zhang, X. Huang and R. Zhu, Chem. Commun., 2011, 47, 10650-10652.

28 Y. Dong, N. Zhou, X. Lin, J. Lin, Y. Chi and G. Chen, Chem. Mater., 2010, 22, 5895-5899.

29 Z. A. Qiao, Y. Wang, Y. Gao, H. Li, T. Dai and Y. Liu, Chem. Commun., 2010, 4, 8812-8814.

30 A. Mandal, A. Dandapat and G. De, Analyst, 2012, 3, 765-772. 31 X. Jin, X. Sun, G. Chen, L. Ding, Y. Li, Z. Liu, Z. Wang, W. Pan, C. Hu and J. Wang, Carbon, 2015, 81, 388-395.

32 X. Gong, Q. Hu, M. C. PDAu, Y. Zhang, S. Shuang, C. Dong and M. M. F. Choi, Nanoscale, 2014, 6, 8162-8170.

33 Z. Q. Xu, L. Y. Yang, X. Y. Fan, J. C. Jin, J. Mei, W. Peng, F. L. Jiang, Q. Xiao and Y. Liu, Carbon, 2014, 66, 351-360.

34 Y. Han, D. Tang, Y. Yang, C. Li, W. Kong, H. Huang, Y. Liu and Z. Kang, Nanoscale, 2015, 7, 5955-5962.

35 Y. Xu, M. Wu, Y. Liu, X. Z. Feng, X. B. Yin, X. W. He and Y. K. Zhang, Chem.-Eur. J., 2013, 19, 2276-2283.

36 S. Liu, J. Tian, W. Lei, Y. Zhang, X. Qin, Y. Luo, A. M. Asiri, A. O. AlYoubi and X. Sun, Adv. Mater., 2012, 24, 2037-2041.

37 X. Sun, C. Brücknerb and Y. Lei, Nanoscale, 2015, 7, 1727817282.
38 S. Li, Y. Li, J. Cao, J. Zhu, L. Fan and X. Li, Anal. Chem., 2014, 86, 10201-10207.

39 X. R. Xu, H. B. Li, J. D. Gu and X. Y. Li, Environ. Toxicol. Chem., 2005, 24, 1310-1314.

40 B. Dhal, H. N. Thatoi, N. N. Das and B. D. Pandey, J. Hazard. Mater., 2013, 250-251, 272-291.

41 S. Chen, X. Hai, X. W. Chen and J. H. Wang, Anal. Chem., 2014, 86, 6689-6694.

42 H. Zhu, F. Lu, X. C. Wu and J. J. Zhu, Analyst, 2015, 140, 7622-7628.

43 M. Q. Yu, H. Wang, F. Fu, L. Y. Li, J. Li, G. Li, Y. Song, M. T. Swihart and E. Q. Song, Anal. Chem., 2017, 89, 40854090.

44 M. Lin, H. Y. Zou, T. Yang, Z. X. Liu, H. Liu and C. Z. Huang, Nanoscale, 2016, 8, 2999-3007.

45 H. J. Zhang, Y. L. Chen, M. J. Liang, L. F. Xu, S. D. Qi, H. L. Chen and X. G. Chen, Anal. Chem., 2014, 86, 9846-9852.

46 J. Qin, Q. Nan, J. Yang and R. Yang, Spectrochim. Acta, Part A, 2018, 203, 421-427.

47 C. X. Zhao, Y. Jiao, F. Hu and Y. L. Yang, Spectrochim. Acta, Part A, 2018, 190, 360-367.

48 J. Shen, S. M. Shang, X. Y. Chen, D. Wang and Y. Cai, Sens. Actuators, B, 2017, 248, 92-100.

49 H. Y. Zhang, Y. Wang, S. Xiao, H. Wang, J. H. Wang and L. Feng, Biosens. Bioelectron., 2017, 87, 46-52.

50 X. J. Gong, Y. Liu, Z. H. Yang, S. M. Shuang, Z. Y. Zhang and C. Dong, Anal. Chim. Acta, 2017, 968, 85-96.

51 V. K. Singh, V. Singh, P. K. Yadav, S. Chandra, D. Bano, V. Kumar, B. Koch, M. Talat and S. H. Hasan, New J. Chem., 2018, 42, 12990-12997.

52 J. L. Chen, X. P. Yan, K. Meng and S. F. Wang, Anal. Chem., 2011, 83, 8787-8793.

53 K. Qu, J. Wang, J. Ren and X. Qu, Chem.-Eur. J., 2013, 19, 7243-7249.

54 D. Zhao, H. Song, L. Hao, X. Liu, L. Zhang and Y. Lv, Talanta, 2013, 107, 133-139. 